\title{
Risk Analysis of Supply Chain Finance under Blockchain Technology -- Based on AHP-FCM Model
}

\author{
Xie Wantong ${ }^{1, *}$, Li Yuanming ${ }^{1}$ \\ ${ }^{1}$ International Business School, Dongbei University of Finance and Economics, Shenyang, Liaoning province, China
}

\begin{abstract}
Applying blockchain technology to supply chain finance is the only way for the in-depth development of supply chain finance. However, increasingly prominent risk issues restrict the stable development of blockchain finance, so it is necessary to identify and analyze supply chain financial risks under blockchain technology. This article summarizes the six aspects of macro-industry analysis, credit risk, supply chain relationship risk, pledge risk, operational risk, and blockchain system risk from the internal and external influencing factors for risk identification, and uses fuzzy cognitive maps and hierarchical analysis. Based on the analysis of supply chain financial risks under blockchain technology, the results show that the three indicators of supply chain relationship risk, operational risk and blockchain system risk are relatively high risk, and have the greatest impact on supply chain financial risks.
\end{abstract}

\section{The introduction}

Blockchain technology has greatly improved the efficiency and security of supply chain finance model due to its obvious characteristics of decentralization, immutability and no third party [1 2]. Blockchain finance is not only the necessary stage for the in-depth development of supply chain finance, but also the inevitable result of the extension of blockchain model [3]. However, in the context of incomplete blockchain technology and imperfect laws and regulations, a series of risk problems are restricting the stable development of supply chain finance under blockchain technology [4]. Therefore, it is particularly important to identify and analyze the risks of Internet finance under the blockchain technology. In terms of risk assessment, literature [5 7] constructed the risk factors of blockchain financial risk assessment system, including policy and legal risks, technical risks, operational risks, credit risks, liquidity risks, etc., and identified the relative importance of various risks. Literature [8 9] introduced block technology to build a decentralized crowdfunding platform, and put forward risk prevention countermeasures.

In conclusion, under the existing block chain technology of supply chain finance risk related research mainly focused on the application prospect and the existing regulatory issues, and related Suggestions are put forward, with qualitative analysis is given priority to, subjectivity is stronger, but for block chain technology under the supply chain is a rare special research literature of financial risk, cause the limited scope of risk identification, risk prevention countermeasures for the lack of reliability. Therefore, based on the block chain technology under the supply chain financial risk as the research object, and analytic hierarchy process (ahp) combined with the fuzzy cognitive map, from the macroscopic industry analysis, credit risk, the relationship between the supply chain risk, collateral risk, operation risk, chain system risk that six aspects to the block chain technology under the supply chain financial risk analysis, to promote the application and promotion of block chain technology in supply chain finance.

\section{Basic connotation and risk identification of supply chain finance under block chain technology}

\subsection{The basic connotation of supply chain finance under block chain technology}

\subsubsection{Blockchain}

Blockchain is a kind of data structure that uses specific cryptographic mathematical algorithms to encrypt each transaction information and record it in a data block (consisting of 62 characters, 52 English lowercase letters and 10 Arabic numerals) and connect them together in a chronological chain structure. Blockchain uses distributed multi-points to record and update data blocks, and specific cryptographic algorithms to ensure the security of data blocks. It is a new expression of distributed data storage, point-to-point transmission, consensus mechanism, encryption algorithm and other technologies. In essence, it is a central-free database.

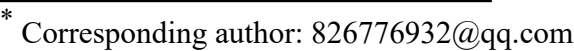




\subsubsection{Blockchain features}

(1) Decentralization and consensus mechanism. Decentralization is the core feature of blockchain technology. In blockchain, there is no strong central node to make rules, unify bookkeeping and maintain ledgers. Bookkeeping rules are open (the so-called consensus mechanism), which has lower cost and higher data security compared to the system with a central structure.

(2) immutability. In the blockchain, no subject can delete or tamper with any information.

(3) Accessibility. Block storage information can be easily accessed by any principal.

(4) No third party. Blockchain itself is a platform, thanks to its decentralized nature, no matter what task activities are carried out on the platform, there is no need for the approval of a third party.

\subsubsection{Blockchain supply chain finance}

Chain of supply chain finance refers to the block to the chain of supply chain under the support money trading platform, cross-border payment channels, financing mode, and other financial activities collectively, so that they can achieve end-to-end information transparency, data realtime credit decision, equal participation, data sharing, mass collaboration, will form a complete fluency and realtime information flow can be traced back.

\subsection{Financial risk identification of supply chain under block chain technology}

\subsubsection{External influencing factors}

(1) Macroeconomic environmental factors. If the economic environment is on the rise, the business conditions of small and medium-sized enterprises will be relatively good. On the contrary, if the business conditions of small and medium-sized enterprises are poor, their risks will be increased.

(2) Blockchain policy factors. A series of industrial structure adjustment policies issued by the state will greatly affect the development of small and medium-sized enterprises.

(3) The development stage of the industry. If the industry has a good development prospect, then the business situation of small and medium-sized enterprises is in an upward trend, and the overall business risk will be low; on the contrary, the industry is in a decline period, and the business risk is high.

(4) Industry competition intensity factors. If the competition intensity is high in the supply chain, it indicates that the small and medium-sized enterprises have fierce competition for scarce resources, and the positions of the enterprises at the edge of the block will change dramatically, resulting in a significant increase in the instability of the supply chain.

\subsubsection{Internal Influencing Factors}

(1) Risks existing within each participating enterprise. Including the financing enterprise debt paying ability level, financing enterprise quality level, financing enterprise development potential level, core enterprise credit status and core enterprise profitability.

(2) Risk factors of banks. In the process of approval of the financing process, the bank may have inaccurate evaluation of the value of pledges.

(3) Risk factors of third-party logistics enterprises. If the supervision of third-party logistics is not in place, it will cause the price of the pledges to be devaluated to the extent that they cannot cover the financing amount when the financing deadline arrives, which is easy to cause default behavior, which will cause losses to the bank and generate risks.

(4) Relationship risk factors between enterprises. Mainly, there are risks in the relationship between various actors involved in supply chain finance on the blockchain.

(5) Risks of blockchain system. In the application and implementation of blockchain technology, we should also consider the security, reliability, stability and other risks brought by the introduction of the general system to enterprises.

\section{The research methods}

\subsection{Fuzzy cognitive map}

Fuzzy cognitive map (FCM) predicts the state of the system at different stages through index nodes and correlation matrix. It has strong reasoning ability and feedback mechanism, can quantify research problems, and provide reliable analysis for the interaction between index nodes of supply chain financial risk under blockchain technology.

The fuzzy cognitive graph model of supply chain financial risk under blockchain technology can be represented by $G=(C, E, W, f, A)$. Where, $C=$ $\left\{c_{1}, c_{2}, \ldots, c_{n}\right\}$ is composed of $\mathrm{n}$ index nodes, $E=$ $\left\{\left\langle c_{i}, c_{j}\right\rangle \mid c_{i}, c_{j} \in C\right\}$ is composed of the correlation arc in $\mathrm{C}$, where the correlation arc $\left\langle c_{i}, c_{j}\right\rangle$ refers to the correlation relationship between $c_{i}$ and $c_{j}, W=$ $\left\{w_{i j} \mid w_{i j}\right.$ is the weight of the associated arc < $\left.c_{i}, c_{j}>\right\}$, and $w_{i j} \in[-1,1]$ is the causal weight between index section $c_{i}$ and $c_{j}$. When $w_{i j}>0$, it indicates that $c_{i}$ has positive effect on $c_{j}$. When $w_{i j}<0$, it indicates that $c_{i}$ has negative effect on $c_{j}$; When $w_{i j}=0$, it indicates that $c_{i}$ has no effect on $c_{j} . f$ is the threshold function, which can convert the index state value to the interval $[0,1]$. Sigmoid function is usually adopted, as shown in Equation (1). A is used to represent the state value of the indicator node, then the state value of the fuzzy cognitive graph $\mathrm{G}$ at time $\mathrm{t}$ is $A_{G^{(t)}}=\left(A_{c_{1}(t)}, A_{c_{2}(t)}, \ldots, A_{c_{n}{ }^{(t)}}\right)$, where the state value $A_{c_{1}}(t) \in[-1.1]$ represents the effect of the indicator node on the system at time t. Equation (2) shows the fuzzy reasoning formula: 


$$
\begin{gathered}
f(x)=\frac{1}{1+e^{-\alpha x}} \\
A_{c_{i}(t+1)}=f\left(A_{c_{i}(t)}+\sum_{j=1, i \neq j}^{n} A_{c_{j}(t)} \cdot w_{i j}\right)
\end{gathered}
$$

In the formula, $A_{c_{i}}(t) 、 A_{c_{j}}(t)$ are the state values of index nodes $c_{i}, c_{j}$ at time t, respectively. By inputting the initial state value $A_{G^{(0)}}$, through repeated iterative calculation of $f$, the system will enter the steady state when the system falls into a circular state, and the calculation will end at this time.

\subsection{Analytic hierarchy process}

The key to the construction of the fuzzy cognitive map model of supply chain financial risks under blockchain technology is to obtain the association matrix. In this paper, the Analytic Hierarchy Process (AHP) is introduced into FCM, and the specific steps are as follows:

(1) According to the identified risks of supply chain finance on the blockchain, the principle of analytic hierarchy process is adopted to construct the risk hierarchy structure. Figure 1 is the risk hierarchy structure model of supply chain finance on the blockchain.

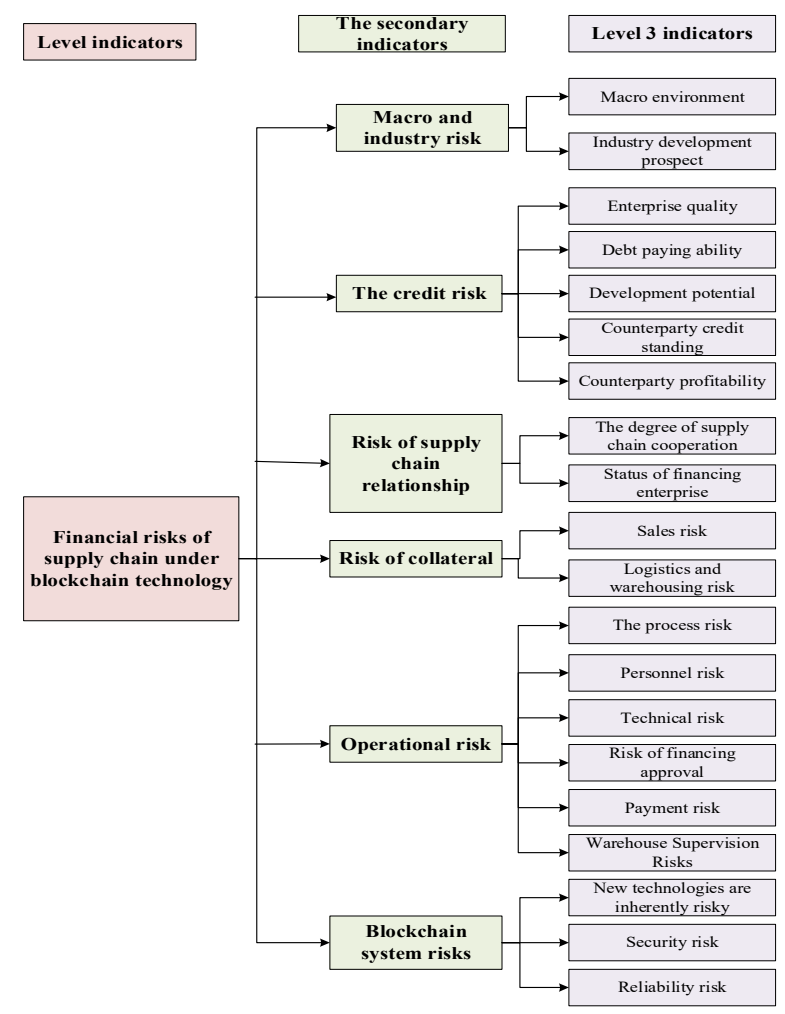

Fig. 1. Risk hierarchy model of supply chain finance on blockchain.

(2) The 1-9 scale method was used to compare the importance of indicators among themselves and construct a judgment matrix $A=\left(a_{i j}\right)_{n \times n}$.

(3) The root method is used to calculate the weight w of supply chain financial risk index under blockchain technology. Taking the index weight $l_{1}$ as an example, $w_{l_{1}}$ can be expressed as:

$$
w_{l_{1}}=\frac{\left(\prod_{j=1}^{n} a_{1 j}\right)^{1 / n}}{\sum_{i=1}^{n}\left(\prod_{j=1}^{n} a_{i j}\right)^{1 / n}}
$$

(4) Consistency test can avoid the random characteristic of the judgment matrix without losing the significance of judgment. Specifically, it can be expressed as:

$$
\begin{gathered}
C I=\alpha_{\max }-\frac{n}{n-1} \\
C R=\frac{C I}{R I}
\end{gathered}
$$

Where, $\alpha_{\max }$ is the maximum eigenvalue, $C I$ is the consistency index, $R I$ is the average random consistency index, and $C R$ is the consistency ratio. When $C R<0.1$, it passes the test.

\section{The example analysis}

In the form of questionnaire and expert consultation, this paper inquired about the opinions of relevant practitioners, and obtained the relative importance of each index by referring to the weight of traditional supply chain risk index. Then, through 1-9 scale method, the weight value of each index of supply chain financial risk under blockchain technology was calculated by Equation (3).

Table 1. The weight of various indicators of supply chain finance under blockchain technology.

\begin{tabular}{|c|c|c|c|}
\hline $\begin{array}{c}\text { The } \\
\text { index } \\
\text { code }\end{array}$ & Weight value & $\begin{array}{c}\text { The index } \\
\text { code }\end{array}$ & Weight value \\
\hline$l_{1}$ & 0.0701 & $l_{31}$ & 0.3333 \\
\hline$l_{2}$ & 0.1502 & $l_{32}$ & 0.6667 \\
\hline$l_{3}$ & 0.2102 & $l_{41}$ & 0.3333 \\
\hline$l_{4}$ & 0.1238 & $l_{42}$ & 0.6667 \\
\hline$l_{5}$ & 0.1792 & $l_{51}$ & 0.1556 \\
\hline$l_{6}$ & 0.2664 & $l_{52}$ & 0.1950 \\
\hline$l_{11}$ & 0.2500 & $l_{53}$ & 0.1895 \\
\hline$l_{12}$ & 0.7500 & $l_{54}$ & 0.1132 \\
\hline$l_{21}$ & 0.0741 & $l_{55}$ & 0.1166 \\
\hline$l_{22}$ & 0.1994 & $l_{56}$ & 0.2536 \\
\hline$l_{23}$ & 0.1201 & $l_{61}$ & 0.2536 \\
\hline$l_{24}$ & 0.2726 & $l_{62}$ & 0.2536 \\
\hline$l_{25}$ & 0.3339 & $l_{63}$ & 0.4531 \\
\hline
\end{tabular}

Table 4 for block chain technology under the supply chain financial indicators weight, by table 4 , the relationship of supply chain risk, operation risk and chain block system these three indicators risk is higher, also is the biggest impact on the financial risk of supply chain, the chain block system for the biggest risk, and the chain of chain system risk in the block stability and reliability of the biggest risk. This is also in line with the current development environment of blockchain technology. The 
main reason is that the blockchain technology is in the early stage of development, the equipment of the blockchain supply chain lacks independent intellectual property rights, and there are no perfect laws and regulations to escort it.
Taking the judgment matrix of $I_{3}$ as an example, the consistency test was carried out, and $\alpha_{\max }=5.21, \mathrm{n}=5$, $C I=0.053, R I=1.12, C R=0.047<0.10$, that is, the consistency test of each judgment matrix can be obtained by applying the above process to each judgment matrix through the test, as shown in Table 2.

Table 2. Consistency test of judgment matrix.

\begin{tabular}{|c|c|c|c|c|c|l|}
\hline $\begin{array}{c}\text { Judgment matrix } \\
\text { code }\end{array}$ & $\alpha_{\max }$ & $\mathrm{n}$ & $C I$ & $R I$ & $C R$ & $\begin{array}{c}\text { Whether it passes } \\
\text { the test }\end{array}$ \\
\hline$l$ & 6.2462 & 6 & $0.049<0.10$ & 1.24 & $0.040<0.10$ & yes \\
\hline$l_{1}$ & 2.00 & 2 & $0.000<0.10$ & 0.00 & $0.000<0.10$ & yes \\
\hline$l_{2}$ & 5.21 & 5 & $0.053<0.10$ & 1.12 & $0.047<0.10$ & yes \\
\hline$l_{3}$ & 2.00 & 2 & $0.000<0.10$ & 0.00 & $0.000<0.10$ & yes \\
\hline$l_{4}$ & 2.00 & 2 & $0.000<0.10$ & 0.00 & $0.000<0.10$ & yes \\
\hline$l_{5}$ & 6.42 & 6 & $0.085<0.10$ & 1.24 & $0.069<0.10$ & yes \\
\hline$l_{6}$ & 3.05 & 3 & $0.027<0.10$ & 0.58 & $0.046<0.10$ & yes \\
\hline
\end{tabular}

As can be seen from Table 2, the financial risk indicators of the supply chain under the blockchain technology all pass the consistency test required in the analytic hierarchy process.

\section{Conclusion}

In this paper, based on the basic connotation of supply chain finance under the block chain technology, from the internal and external factors affecting the analysis, sums up the macro industry credit risks, risks of supply chain relationship, the pledge risk, operation risk, system risk chain blocks, these six aspects, and has carried on the risk identification, using the analytic hierarchy process (ahp) and fuzzy cognitive map to each index weight assignment and index score. The final risk value of each index is calculated, which effectively identifies the unique risk points of the supply chain system on the blockchain.

\section{References}

1. Baykar Silahli, Kemal Dincer Dingec, Atilla Cifter, et al. Portfolio value-at-risk with two-sided Weibull distribution: Evidence from cryptocurrency markets[J]. Finance Research Letters, 2019, :101425101425.

2. Kowalski Michał, Lee Zach W.Y., Chan Tommy K.H.. Blockchain technology and trust relationships in trade finance $[\mathrm{J}]$. Technological Forecasting and Social Change, 2021, 166

3. Valentina Gatteschi, Fabrizio Lamberti, Claudio Demartini, et al. Blockchain and Smart Contracts for Insurance: Is the Technology Mature Enough?[J]. Future Internet, 2018, 10(2):20-20.

4. M Kassou, S Bourekkadi, S Khoulji, et al. Blockchain-based medical and water waste management conception[J]. E3S Web of Conferences, 2021, 234:00070-.
5. Li Z., Zhong Ray Y., Tian Z.G., et al. Industrial Blockchain: A state-of-the-art Survey[J]. Robotics and Computer-Integrated Manufacturing, 2021, 70

6. Singh P. Blockchain based Security Solutions with IoT Application in Construction Industry[J]. IOP Conference Series: Earth and Environmental Science, 2020, 614(1):012052-.

7. Jacob Lohmer, Niels Bugert, Rainer Lasch. Analysis of resilience strategies and ripple effect in blockchain-coordinated supply chains: An agentbased simulation study[J]. International Journal of Production Economics, 2020, 228:107882-107882.

8. Darja Milic. The impact of non-banking financial institutions on monetary policy transmission in Euro area[J]. Empirical Economics, 2020, :1-39.

9. Francisco-Javier Canto-Cuevas, María-José PalacínSánchez, Filippo Di Pietro. Trade Credit as a Sustainable Resource during an SME's Life Cycle[J]. Sustainability, 2019, 11(3):670. 